International Symposium on Unified Symmetry: In the Small and in the

Large, Miami, FL, 2/2-5/95.

\title{
ACCELERATING POLARIZED BEAMS AT THE AGS *
}

\author{
Thomas Roser \\ AGS Department \\ Brookhaven National Laboratory \\ Upton, N.Y. 11973, USA
}

RECEIVED

NOV 291995
OSTI

\section{INTRODUCTION}

The acceleration of polarized beams in circular accelerators is complicated by the presence of numerous depolarizing resonances. During acceleration, a depolarizing resonance is crossed whenever the spin precession frequency equals the frequency with which spinperturbing magnetic fields are encountered. There are two main types of depolarizing resonances corresponding to the possible sources of such fields: imperfection resonances, which are driven by magnet errors and misalignments, and intrinsic resonances, driven by the focusing fields.

The resonance conditions are usually expressed in terms of the spin tune $\nu_{s}$, which is defined as the number of spin precessions per revolution. For an ideal planar accelerator, where orbiting particles experience only the vertical guide field, the spin tune is equal to $G \gamma[1]$, where $G=1.7928$ is the anomalous magnetic moment of the proton and $\gamma$ is the relativistic Lorentz factor. The resonance condition for imperfection depolarizing resonances arise when $\nu_{s}=G \gamma=n$, where $n$ is an integer. Imperfection resonances are therefore separated by only $523 \mathrm{MeV}$ energy steps. The condition for intrinsic resonances is $\nu_{s}=G \gamma=k P \pm \nu_{y}$, where $k$ is an integer, $\nu_{y}$ is the vertical betatron tune and $P$ is the superperiodicity. For the AGS, $P=12$ and $\nu_{y} \approx 8.8$. For most of the time during the acceleration cycle, the precession direction, or stable spin direction, coincides with the main vertical magnetic field. Close to a resonance, the stable spin direction is perturbed away from the vertical direction by the resonance driving fields. When a polarized beam is accelerated through an isolated resonance, the final polarization can be

$$
P_{f} / P_{i}=2 e^{-\frac{\pi \mid l^{2}}{2 \alpha}}-1,
$$

where $P_{i}$ and $P_{f}$ are the polarizations before and after the resonance crossing, respectively, $\epsilon$ is the resonance strength obtained from the spin rotation of the driving fields, and $\alpha$ is the 


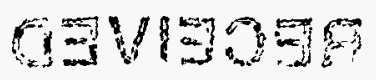

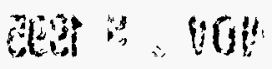

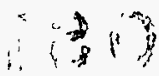

\section{DISCLAIMER}

This report was prepared as an account of work sponsored by an agency of the United States Government. Neither the United States Government nor any agency thereof, nor any of their employees, makes any warranty, express or implied, or assumes any legal liability or responsibility for the accuracy, completeness, or usefulness of any information, apparatus, product, or process disclosed, or represents that its use would not infringe privately owned rights. Reference herein to any specific commercial product, process, or service by trade name, trademark, manufacturer, or otherwise does not necessarily constitute or imply its endorsement, recommendation, or favoring by the United States Government or any agency thereof. The views and opinions of authors expressed herein do not necessarily state or reflect those of the United States Government or any agency thereof. 


\section{DISCLAIMER}

Portions of this document may be illegible in electronic image products. Images are produced from the best available original document. 


\section{E880 Partial Snake Test at the AGS}

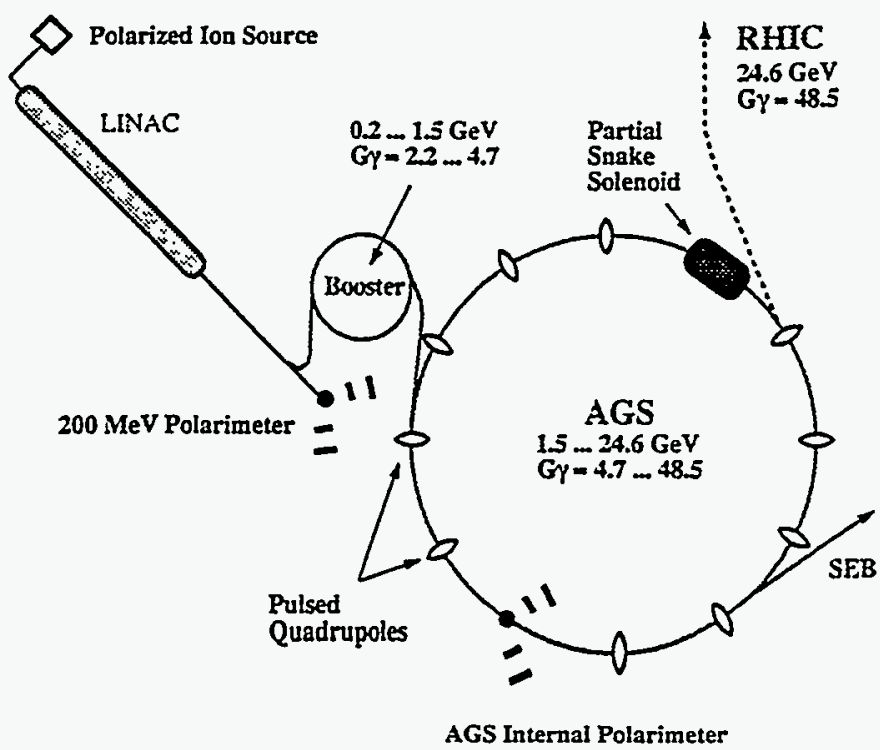

Figure 1: Layout of the AGS accelerator complex showing the location of the Partial Siberian Snake, the pulsed quadrupoles, and the AGS internal polarimeter

change of the spin tune per radian of the orbit angle. When the beam is slowly $\left(\alpha \ll|\epsilon|^{2}\right)$ accelerated through the resonance, the spin vector will adiabatically follow the stable spin direction resulting in spin flip. However, for a faster acceleration rate partial depolarization or partial spin flip will occur. Traditionally, the intrinsic resonances are overcome by using a betatron tune jump, which effectively makes $\alpha$ large, and the imperfection resonances are overcome with the harmonic corrections of the vertical orbit to reduce the resonance strength $\epsilon$ [3]. At high energy, these traditional methods become difficult and tedious.

By introducing a 'Siberian Snake' [4], which is a $180^{\circ}$ spin rotator of the spin about a horizontal axis, the stable spin direction remains unperturbed at all times as long as the spin rotation from the Siberian Snake is much larger than the spin rotation due to the resonance driving fields. Therefore the beam polarization is preserved during acceleration. Such a spin rotator can be constructed by using either solenoidal magnets or a sequence of interleaved horizontal and vertical dipole magnets producing only a local orbit distortion. Since the orbit distortion is inversely proportional to the momentum of the particle, such a dipole snake is particularly effective for high-energy accelerators, e.g. energies above about $30 \mathrm{GeV}$. For lower-energy synchrotrons, such as the Fermilab booster and the Brookhaven AGS with weaker depolarizing resonances, a partial snake[5], which rotates the spin by less than $180^{\circ}$, is sufficient to keep the stable spin direction unperturbed at the imperfection resonances.

\section{AGS PARTIAL SIBERIAN SNAKE TESTS}

Two polarized beam test runs of experiment E- 880 at the AGS have recently demonstrated the feasibility of polarized proton acceleration using a $5 \%$ partial Siberian Snake. During the first run[6] in April 1994 it was shown that a $5 \%$ Snake is sufficient to avoid depolarization due to the imperfection resonances without using the harmonic correction method. Fig. 2 shows the evolution of the beam polarization as the beam energy and therefore $G \gamma$ is 


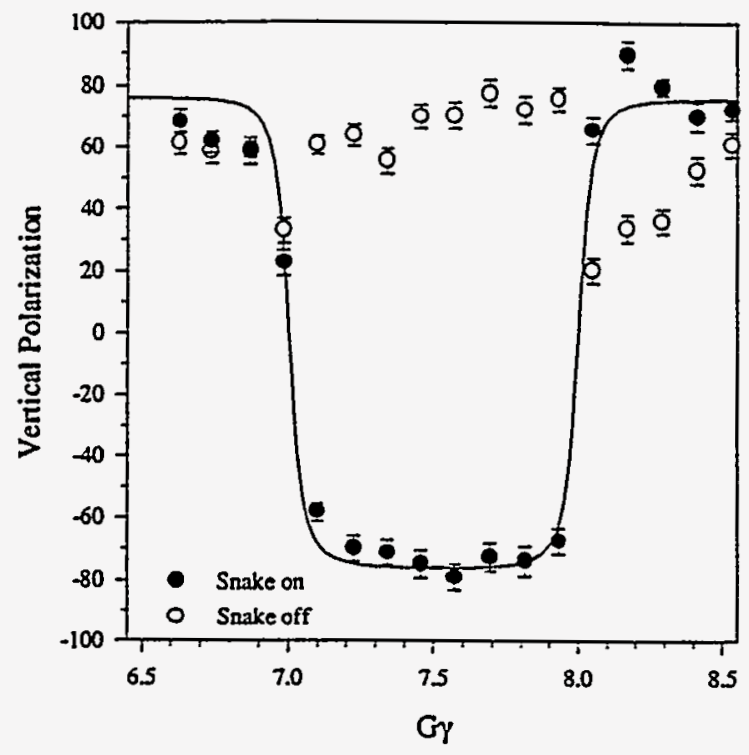

Figure 2: The measured vertical polarization as a function of the spin tune $G \gamma$ for a $10 \%$ snake is shown with and without a snake. Note here that partial depolarization at $G \gamma=8$ is avoided by using a $10 \%$ snake. The solid line is the predicted energy dependence of the polarization.

increased. As predicted the polarization reverses the sign whenever $G \gamma$ is equal to an integer.

Fig. 3 shows the achieved polarization as a function of beam energy. It shows that no polarization was lost at the imperfection resonances. The only polarization loss occurred at the location of the intrinsic resonances for which the pulsed quadrupoles are required for the tune jump method. During the first run the pulsed quadrupoles were not available. During the second run in December 1994 it was shown that it is possible to use the tune jump method in the presence of the partial Snake. A new record energy for accelerated polarized beam of $25 \mathrm{GeV}$ was reached with about $12 \%$ beam polarization left. Again no polarization was lost due to the imperfection resonances and depolarization from most intrinsic resonances was avoided with the tune jump quadrupoles. However, as can be seen from Fig. 3, significant amount of polarization was lost at $G \gamma=0+\nu_{y}, 12+\nu_{y}$ and $G \gamma=36+\nu_{y}$. The first two of these three resonances were successfully crossed previously and it will require further study to explain the unexpected polarization loss. The strength of the tune jump quadrupoles is not sufficient to jump the last resonance. We attempted to induce spin flip at this resonance but were only partially successful. During the next study run the method of inducing spin flip at intrinsic resonances will be further investigated[7].

\section{TOWARDS A POLARIZED PROTON COLLIDER}

With the successful tests of Siberian Snakes the stage is set for the acceleration of polarized proton beams to much higher energies to be used in collider experiments to explore spin effects at the highest energies attainable. Polarized protons from the Brookhaven AGS will be injected into the two RHIC rings to allow for up to $\sqrt{s}=500 \mathrm{GeV}$ collisions with both beams polarized. With full Siberian Snakes all depolarizing resonances should be avoided 


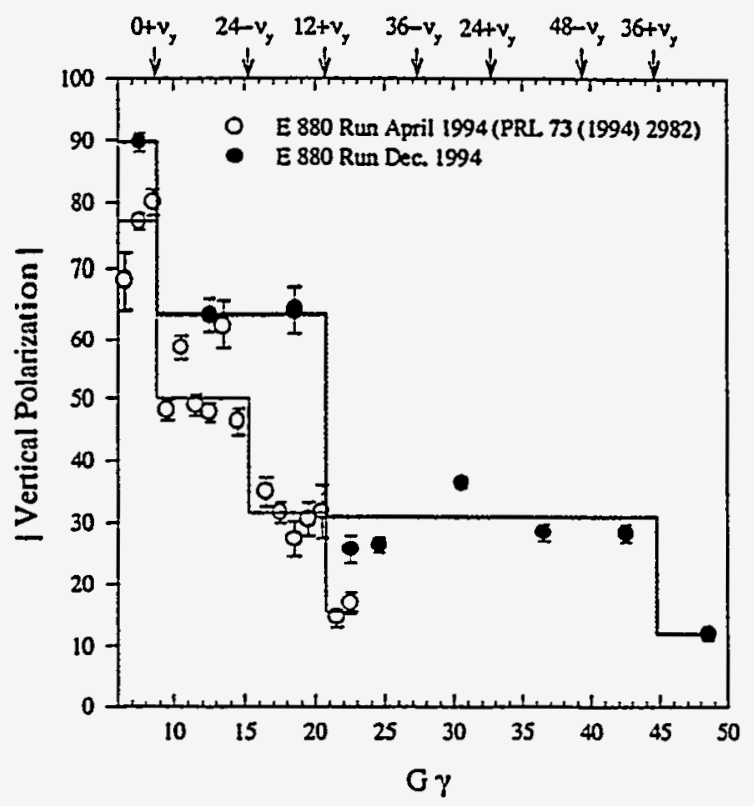

Figure 3: The measured absolute value of the vertical polarization at $G \gamma=n+\frac{1}{2}$ up to $G \gamma=48.5$ which corresponds to an energy fo $25 \mathrm{GeV}$. The partial depolarization is due to intrinsic spin resonances at $G \gamma$ values indicated at the top of the figure. The results from the Dec. 1994 run are preliminary.

since the spin tune is a half-integer independent of energy. However, if the spin disturbance from small horizontal fields is adding up sufficiently between the Snakes depolarization can still occur. This is most pronounced when the spin rotation from all the focusing filed add up coherently which is the case at the strongest intrinsic resonances. At RHIC two Snakes can still cope with the strongest intrinsic resonance whereas at Tevatron energies six Snakes would be needed.

Of particular interest is the design of the Siberian Snakes (two for each ring) and the spin rotators (four for each collider experiment) for RHIC. Proposed by V. Ptitsin and Yu. Shatunov from BINP[8], it is based on helical dipole magnet modules each having a full 360 degree helical twist. Using helical magnets minimizes orbit excursions which is most important at injection energy. Fig. 4 shows the aluminum former for the prototype helical dipole magnet now under construction at Brookhaven. The construction of a large bore high field helical dipole presents a formidable challenge for present superconducting magnet technology.

\section{ACKNOWLEDGMENT}

This work was performed under the auspices of the U.S. Department of Energy.

\section{REFERENCES}

[1] L.H. Thomas, Phil. Mag. 3, 1 (1927); V. Bargmann, L. Michel, V.L. Telegdi, Phys. Rev. Lett. 2, 435 (1959). 


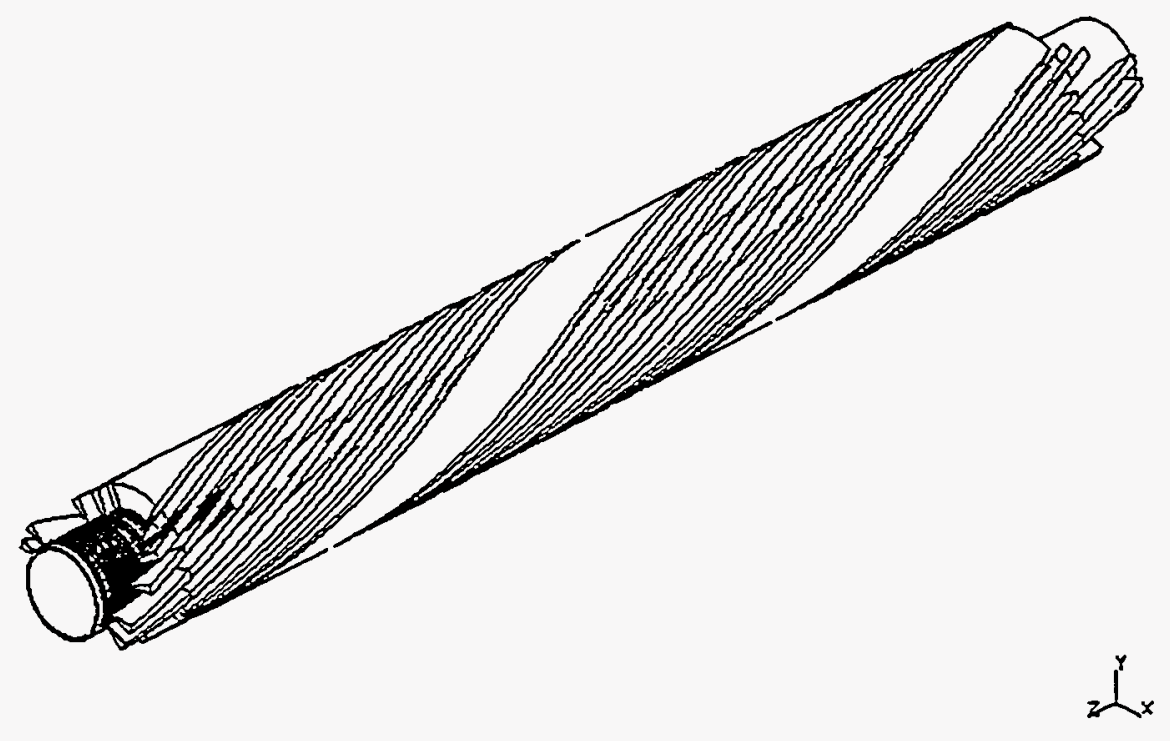

Figure 4: Aluminum former for the helical dipol magnet prototype under construction at BNL

[2] M. Froissart and R. Stora, Nucl. Instr. Meth., 1, 297 (1960).

[3] T. Khoe et al., Part. Accel. 6, 213 (1975); J.L. Laclare et al., J. Phys. (Paris), Colloq. 46, C2-499 (1985); H. Sato et al., Nucl. Inst. Meth., Phys. Res. Sec A272, 617 (1988); F.Z. Khiari, et al., Phys. Rev. D39, 45 (1989).

[4] Ya.S. Derbenev et al., Part. Accel. 8, 115 (1978).

[5] T. Roser, AIP Conf. Proc. No. 187, ed. K.J. Heller p.1442 (AIP, New York, 1988).

[6] H.Huang et al., Phys. Rev. Lett. 73, 2982 (1994)

[7] T. Roser, in Proc. of the 10th Int. Symp. on High Energy Spin Physics, Nagoya, Japan, p. 429 (1992).

[8] V.I.Ptitsin and Yu.M.Shatunov, Helical Spin Rotators and Snakes, Proc. Third Workshop on Siberian Snakes and Spin Rotators (A.Luccio and T.Roser Eds.) Upton, NY, Sept. 12-13,1994, Brookhaven National Laboratory Report BNL-52453, p.15 